\title{
A Theorem on Asymptotic Expansion of Feynman Amplitudes
}

\author{
M. C. Bergère ${ }^{1}$, C. de Calan², and A. P. C. Malbouisson ${ }^{2 \star}$ \\ 1 Service de Physique Théorique, CEN Saclay, F-91190 Gif-sur-Yvette, France \\ 2 Centre de Physique Théorique, Ecole Polytechnique, F-91128 Palaiseau, France
}

\begin{abstract}
For any Feynman amplitude, where any subset of invariants and/or squared masses is scaled by a real parameter $\lambda$ going to zero or infinity, the existence of an expansion in powers of $\lambda$ and $\ln \lambda$ is proved, and a method is given for determining such an expansion. This is shown quite generally in euclidean metric, whatever the external momenta (exceptional or not) and the internal masses (vanishing or not) may be, and for some simple cases in minkowskian metric, assuming only finiteness of the - eventually renormalized - amplitude before scaling. The method uses what is called "Multiple Mellin representation", the validity of which is related to a "generalized powercounting" theorem.
\end{abstract}

\section{Introduction}

In this paper we give a mathematical method for studying the asymptotic behaviour of Feynman amplitudes, that is integrals $G\left(a_{k}\right)$ corresponding to given Feynman graphs. Notation $\left\{a_{k}\right\}$ represents the set of invariants $\left(\sum p\right)^{2}$ built from external momenta $p$, and of internal squared masses $m_{i}^{2}$. By asymptotic behaviour we mean an expansion in a real parameter $\lambda$ scaling some $a_{k}$, s, say $\left\{a_{m}\right\}$, the other ones, say $\left\{a_{n}\right\}$, remaining fixed. Conventionally we take $\lambda$ as going to infinity, but of course the method applies as well to the case of invariants or masses going to zero, by dimensional argument:

$$
G\left(a_{m}, \frac{1}{\lambda} a_{n}\right)=\lambda^{\omega} G\left(\lambda a_{m}, a_{n}\right)
$$

We emphasize that our method is quite general, since it applies to any asymptotic limit (any choice of the subset $\left\{a_{m}\right\}$ ), for arbitrarily given external momenta, generic or exceptional, and for arbitrary finite or vanishing masses. In this paper we consider for simplicity only scalar particles with non-derivative couplings. Then for any Feynman amplitude in Euclidean metric (and for some

* On leave of absence from University of Bahia (Brazil). Fellow of CAPES, Brazıl 
simple situations in Minkowskian metric), our main result is to establish the following:

Theorem. $G\left(\lambda a_{m}, a_{n}\right)$ has an asymptotic expansion of the type:

$$
G\left(\lambda a_{m}, a_{n}\right)=\sum_{p=p_{\max }}^{-\infty} \lambda^{p} \sum_{q=0}^{q_{\max }(p)} \ln ^{q} \lambda g_{p q}\left(a_{m}, a_{n}\right),
$$

where $p$ runs over the rational values of a decreasing arithmetic progression, with $p_{\max }$ as "leading power", and $q$, for a given $p$, runs over a finite number of nonnegative integer values.

Such a theorem was proved in the past by Weinberg [1] for a specific kind of asymptotic behaviour: scaling by $\lambda$ of all external momenta of a convergent Euclidean amplitude; then the asymptotic expansion (I.2) is such that $p$ runs only over integer values, and $p_{\max }$ is determined. Later Fink [2] found $q_{\max }\left(p_{\max }\right)$, and also, the theorem was extended to divergent renormalized amplitudes [3].

It was the search for a proof of the "power counting" rule in the case of a general asymptotic limit which motivated our work. We went back to the essence of the Bogoliubov-Parasiuk-Hepp renormalization theory, and abstracted from it the notion of FINE polynomials (see Sect. II). Combining this with the use of the multiple Mellin representation (see Sect. III), we were able to prove Theorem (I.2) very generally, even in the cases where "naive" power counting does not work.

In the asymptotic problem $s \rightarrow \infty$ (Regge pole behaviour of four bodies amplitudes) this theorem had not been established before. Several papers [4] deal with the determination of $p_{\max }$ and $q_{\max }\left(p_{\max }\right)$ and a general rule was given by Zavyalov and Stepanov [5], though their proof relies on "naive" power counting which is not a priori correct in this kind of asymptotic behaviour. For graphs which occur in low orders of perturbation [6] or for "ladder" graphs [7], $g_{p_{\max } q_{\max }\left(p_{\max }\right)}$ had been determined, but even in these cases the other coefficients $g_{p_{\max } q}$ were not all determined in an explicit way.

Another interesting asymptotic behaviour is the "infra-red" limit of a mass going to zero. In quantum electrodynamics, the on mass-shell amplitudes develop infrared singularities which must exponentiate by a mechanism described in a classical paper by Yennie et al. [8], although no rigorous proof has been yet given for infrared exponentiation and cancellation. Here again, the lack of rigor relies on the fact that the power counting is not valid. More recently Cvitanovic and Kinoshita [9] gave specific rules in the Feynman parameters space, and extracted infrared divergencies by non-linear power counting methods, which can be made rigorous and complete with the use of our present technique. Similarly, the techniques of Grammer Yennie, and of de Rafael and Korthals-Altes [10], allow a summation of infrared logarithms, which becomes rigorous once our Theorem (I.2) is assumed, with $p_{\max }=0$ and $q_{\max }$ bounded by the number of photon lines.

The extension of such results to quantum chromodynamics [11] does not seem to be completely understood yet, and we think that our method would also be useful for its study.

Another application of the multiple Mellin representation is to justify the various manipulations which have to be performed if we wish to compute the 
coefficients $g_{p q}$. For a given asymptotic regime and for the most general graph contributing to the leading power, the coefficients $g_{p_{\max } q}$ are then obtained as integrals attached to subgraphs and reduced graphs, and geometric rules may be given to construct them. These rules may later be used to explain and perform an eventual summation of all logarithms which occur when we sum the contributions to the leading power over all Feynman amplitudes of a vertex function. We achieved such a program in the case of the scaling of all momenta [3, 12], using only single Mellin transform. In the case $s \rightarrow \infty$ for four bodies amplitudes of $\phi^{3}$ field theory, using multiple Mellin techniques from this paper, we have rigorously built the well-known Regge behaviour [13], and we are in process to extend the same program to Regge pole behaviour in $\phi^{4}$ field theory.

Section II gives notations and general comments. The multiple Mellin representation is given in Sect. III and the asymptotic expansion is determined in Sect. IV, for any Feynman amplitude in Euclidean metric. The same results are extended in Sect. V to the case of Minkowskian metric, for some simple situations, and Sect. VI gives a short discussion and examples of application.

\section{Notations and Comments}

\section{Parametric Representation and Mellin Transform}

Given a Feynman graph, $i$ is an index for internal lines, $i=1, \ldots, l$, and $L$ is the number of independent loops. The corresponding amplitude in euclidean metric is defined by the well-known parametric integral representation (see e.g. Ref. [9] and other references therein):

$$
G\left(a_{k}\right)=\int_{0}^{\infty} \prod_{i=1}^{l} d \alpha_{i} \frac{1}{U^{D / 2}\left(\alpha_{i}\right)} e^{-\frac{V\left(a_{k} ; \alpha_{i}\right)}{U\left(\alpha_{i}\right)}}
$$

where $D$ is the space-time dimension and $U$ and $V$ are the Symanzik polynomials:

$$
U\left(\alpha_{i}\right)=\sum_{1 \cdot T} \prod_{i \notin 1 \cdot T} \alpha_{i}
$$

$U\left(\alpha_{i}\right)$ is an homogeneous polynomial with degree $L$. The sum extends over all different "one-trees" $1 \cdot T$ (connected subgraphs, without loop, linking all vertices of the graph).

$$
V\left(a_{k} ; \alpha_{i}\right)=\sum_{2 \cdot T}\left[\left(\prod_{i \notin 2 \cdot T} \alpha_{i}\right)\left(\sum_{2 \cdot T} p\right)^{2}\right]+\left(\sum_{i=1}^{l} \alpha_{i} m_{i}^{2}\right) U\left(\alpha_{i}\right) .
$$

$V\left(a_{k} ; \alpha_{i}\right)$ is an homogeneous polynomial with degree $L+1$, and real positive coefficients $a_{k}=\left(\sum p\right)^{2}, m_{i}^{2}$. The sum extends over all different "two trees" $2 \cdot T$ (subgraphs without loop, with two connected components, linking all vertices of the graph). $\left(\sum_{2 \cdot T} p\right)^{2}$ is the invariant built by squaring the sum of the external momenta over one of the connected components of the two-tree (any one of them equivalently, by momentum conservation). 
Introducing now the scaling parameter $\lambda, V$ is split into two parts:

$$
V\left(\lambda a_{m}, a_{n} ; \alpha_{i}\right)=\lambda W\left(a_{m} ; \alpha_{i}\right)+R\left(a_{n} ; \alpha_{i}\right)
$$

and the behaviour of $G\left(\lambda a_{m}, a_{n}\right)$ for infinite $\lambda$ is related to the vanishing of $W\left(a_{m} ; \alpha_{i}\right)$ when the $\alpha_{i}$ 's vanish. One way for exhibiting this relation is to take, if it exists, the Mellin transform of $G(\lambda)$ (see Ref. [3]), which is nothing but a Fourier-Laplace transform in the variable $\ln \lambda$ :

$$
M(x)=\int_{0}^{\infty} d \lambda \lambda^{-x-1} G(\lambda)=\Gamma(-x) \int_{0}^{\infty} \prod_{i=1}^{l} d \alpha_{i} \frac{1}{U^{D / 2}} e^{-\frac{R}{U}}\left(\frac{W}{U}\right)^{x} .
$$

To the expansion $(I, 2)$ for $G(\lambda)$ correspond meromorphic properties of $M(x)$ :

$$
M(x)=\sum_{p, q} \frac{g_{p q} q !}{(x-p)^{q+1}}
$$

and we are left with the extraction of poles from $M(x)$, that is with the desingularization of the last integral in (II.5). Such a problem has been studied for example in Ref. [14] by appropriate choice of local coordinates, but we shall present in the next sections another method, which we think to be more adapted to explicit computation, interpretation and future reorganization of the coefficients $g_{p q}$.

\section{Hepp's Sectors and "Simultaneous Taylor Series" Property}

One can divide the $\alpha$-integration domain into l! "sectors" [15] by ordering the $\alpha$ 's in all possible ways. To a given permutation $h=\left(i_{1}, \ldots, i_{l}\right)$ of the lines $1, \ldots, l$ corresponds the sector:

$$
\alpha_{i_{1}} \leqq \alpha_{i_{2}} \leqq \ldots \leqq \alpha_{i_{l}}
$$

We choose in this sector new variables $\beta_{i}$ defined by:

$$
\begin{aligned}
\alpha_{i_{l}} & =\beta_{l} \\
\alpha_{i_{l-1}} & =\beta_{l} \beta_{l-1} \\
\alpha_{i_{2}} & =\beta_{l} \beta_{l-1} \ldots \beta_{2} \\
\alpha_{i_{1}} & =\beta_{l} \beta_{l-1} \ldots \beta_{2} \beta_{1} .
\end{aligned}
$$

Then the integration over $\beta_{l}$ can be made explicitly, because the integrands in (II.1) or (II.5) have homogeneity properties, and the remaining integration domain is: $0 \leqq \beta_{i} \leqq 1, i=1, \ldots, l-1$.

Definition. Given some polynomial $P\left(\alpha_{i}\right)$, we call it FINE (essentially Factorized IN Each sector) if in each sector the $\alpha$-ordering induces one and only one dominant monomial in $P$. Algebraically, in the $\beta$-variables, $P$ takes the form:

$$
P\left(\alpha_{i}\right) \rightarrow\left(\prod_{i} \beta_{i}^{r_{i}}\right) Q\left(\beta_{i}\right)
$$


where $Q$ is a polynomial with $Q(0, \ldots, 0) \neq 0$. For example $\alpha_{1}+\alpha_{2}, \alpha_{1} \alpha_{2}+\alpha_{1} \alpha_{3}$ $+\alpha_{2} \alpha_{3}$ are FINE polynomials. Conversely, $\alpha_{1}^{2}+\alpha_{2} \alpha_{3}$ is not FINE as it can be seen in the sector $\alpha_{2} \leqq \alpha_{1} \leqq \alpha_{3}$.

More generally, we speak of a FINE function if in each sector it may be written as:

$$
f\left(\alpha_{i}\right) \rightarrow\left(\prod_{l} \beta_{i}^{x_{i}}\right) g\left(\beta_{i}\right)
$$

where the $x_{i}$, s are arbitrary complex numbers and $g\left(\beta_{i}\right)$ has a simultaneous Taylor expansion around 0 , with non-vanishing constant term $g(0, \ldots, 0) \neq 0$. Arbitrary products of complex powers of FINE functions are FINE functions.

It can be seen from theorems in Ref. [16] that a FINE function $f\left(\alpha_{i}\right)$ has the "simultaneous Taylor series" property: given an arbitrary nest $\mathscr{N}$ (set $\mathscr{N}$ of nested subsets $S$ of lines $i$ ), let us scale each $\alpha_{i}$ by $\prod_{S \ni i} \varrho_{S}$. Then there exist complex powers $z_{S}$ such that $f^{\prime}\left(\varrho_{S} ; \alpha_{i}\right)=\left(\prod_{S \in \mathscr{T}} \varrho_{S}^{z S}\right) f\left(\prod_{S \in i} \varrho_{S} \alpha_{i}\right)$ has a simultaneous Taylor expansion around 0 in the variables $\varrho_{S}$.

In other words the behaviour of $f\left(\alpha_{i}\right)$ around 0 is governed by its vanishing when subsets of $\alpha$-variables go to zero linearly: for a FINE function $f\left(\alpha_{i}\right)$, convergence or divergence of the integral $\int_{0}^{c} \prod_{i} d \alpha_{i} f\left(\alpha_{i}\right)$ can be determined by naive power-counting. For the same reason, eventual divergences in such an integral can be removed by Taylor subtractions [16].

\section{Desingularization of the Mellin Transform}

Desingularization of $M(x)$ in (II.5) is immediately achieved by the change of variables $\alpha \rightarrow \beta$, provided that $U$ and $W$ are FINE polynomials. Now $U$ is always FINE for any graph : that is the reason why Hepp's sectors method works for ultraviolet study of integral (II.1). Also $V$ and $W_{1}=\sum_{2 \cdot T} \prod_{i \notin 2 \cdot T} \alpha_{i}\left(\sum_{2 \cdot T} p\right)^{2}$ are FINE polynomials in euclidean metric: for this reason, the problem of all masses going to zero, in the euclidean case, may be solved in the sectors or by Taylor subtractions [3].

In contrast, neither sums nor parts of FINE polynomials are generally FINE: for many asymptotic problems, the $W$ part of $V$ is not FINE. As an example, $V$ becomes non-FINE for many graphs in quantum electrodynamics, if the photon mass vanishes and if other terms cancel by electron mass-shell condition. Then, as we said, ordinary power-counting becomes wrong: for this reason, in Ref. [9], infrared divergences are explored by non-linear scalings such as $\alpha_{i} \sim 1, \alpha_{j} \sim \varrho$, $\alpha_{k} \sim Q^{2}$.

Such methods of exploration do not constitute a complete proof: to determine an exact asymptotic behaviour, it is in general difficult to characterize the relevant powers of the scaling parameter. We find more efficient to work in the sectors, and to develop another technique explained in the following sections. 


\section{Multiple Mellin Representation}

With the previous notations, for a given Feynman graph ( $l$ lines, $L$ loops) we have an amplitude:

$$
G=\int_{0}^{\infty} \prod_{i=1}^{l} d \alpha_{i} \frac{1}{U^{D / 2}} e^{-\frac{V}{U}}
$$

and we simply assume the existence of this amplitude (before any asymptotic limit), that is the convergence of the integral. The case of ultra-violet divergent graphs will be mentioned at the end of this section.

We first divide $V$ into pieces

$$
V=\sum_{j=1}^{M} V_{j}
$$

in such a way that:

All pieces $V_{j}$ are FINE.

The part $W$ of $V$ which is scaled by $\lambda$ in Sect. IV is a partial sum of $V_{j}^{\prime}$ s:

$$
\begin{aligned}
& W=\sum_{j=1}^{N} V_{j}, \\
& R=\sum_{j=N+1}^{M} V_{j} .
\end{aligned}
$$

This is always possible: at worst, we may divide $V$ into all its monomials. Actually in many physical problems, the splitting of $V$ may be much simpler: see examples in Sect. VI. Even if $V$ is FINE, $W$ and $R$ are generally not; in the particular case where $W$ and $R$ are separately FINE, no further splitting is needed and the method should be equivalent to the simple Mellin transform method of Ref. [3].

Then we decompose $G$ according to Heep's sectors:

$$
G=\sum_{h=1}^{l !} G_{h}
$$

In a given sector $h$, under $\alpha \rightarrow \beta$ change of variables:

$$
\begin{aligned}
& U \rightarrow \beta_{l}^{L}\left(\prod_{i=1}^{l-1} \beta_{i}^{L_{i}}\right) u\left(\beta_{i}\right) \\
& V_{j} \rightarrow \beta_{l}^{L+1}\left(\prod_{i=1}^{l-1} \beta_{i}^{n_{l}}\right) v_{j}\left(\beta_{i}\right) .
\end{aligned}
$$

Because $U$ and $V_{j}$ are homogeneous and FINE, $L_{i}$ and $n_{i j}$ are non-negative integers, $u$ and $v_{j}$ are polynomials in $\beta_{1}, \ldots, \beta_{l-1}$ with real positive coefficients and non-vanishing constant terms. Since $0 \leqq \beta_{i} \leqq 1, u$ and each $v_{j}$ have upper and lower strictly positive bounds. Performing the homogeneity integration over $\beta_{l}$, we 
obtain :

$$
G_{h}=\Gamma(\omega) \int_{0}^{1} \frac{\left(\prod_{i=1}^{l-1} \beta_{i}^{\omega_{t}-1} d \beta_{i}\right) u^{\omega-D / 2}}{\left[\sum_{j=1}^{M} v_{j} \prod_{i=1}^{l-1} \beta_{i}^{n_{t}}\right]^{\omega}}
$$

where $\omega=l-D / 2 L, \omega_{i}=i+(\omega-D / 2) L_{i}$ are integers or half integers. If $V$ is a FINE polynomial, it has in sector $h$ a dominant piece $V_{j_{h}}$. Thus convergence of $G_{h}$ means $\omega_{i}-\omega n_{i j_{h}}>0, i=1, \ldots, l-1$. We effectively recover this condition later. But in the following (having in mind the case of minkowskian metric where $V$ may be nonFINE for exceptional masses or momenta) we consider $V$ as an arbitrary polynomial with positive coefficients.

Using the identity [17]:

$$
\Gamma(x)(A+B)^{-x}=\int_{-\infty}^{+\infty} \frac{d y}{2 i \pi} \Gamma(-\sigma-i y) A^{\sigma+i y} \Gamma(\sigma+i y+x) B^{-\sigma-i y-x}
$$

valid for $\operatorname{Re} x>0, \operatorname{Re} A>0, \operatorname{Re} B>0,-\operatorname{Re} x<\sigma<0$, we get :

$$
G_{h}=\int_{0}^{1}\left(\prod_{i=1}^{l-1} \beta_{i}^{\omega_{l}-1} d \beta_{i}\right) u^{\omega-D / 2} \int_{\sigma_{J}} \prod_{j=1}^{M}\left[\Gamma\left(-z_{j}\right)\left(v_{j} \prod_{i=1}^{l-1} \beta_{i}^{n_{i_{J}}}\right)^{z_{J}}\right],
$$

where

$$
\sum_{j=1}^{M} z_{j}=-\omega ; \quad z_{j}=\sigma_{j}+i y_{j} ; \quad \sigma_{j}<0, \quad j=1, \ldots, M
$$

$\int_{\sigma_{J}}$ is a short notation for $\int_{-\infty}^{+\infty} \frac{d y_{1} \ldots d y_{M-1}}{(2 i \pi)^{M-1}}$.

The problem now is to interchange integrations. This is valid when absolute convergence is verified. But taking the modulus of the integrand we get:

$$
G_{h} \leqq \text { Const }^{t} \int_{0}^{1}\left(\prod_{i=1}^{l-1} \beta_{i}^{\mathrm{Re} \phi_{i}-1} d \beta_{i}\right) \int_{\sigma_{J}} \prod_{j=1}^{M}\left|\Gamma\left(-z_{j}\right)\right|,
$$

where

$$
\operatorname{Re} \phi_{i}=\omega_{i}+\sum_{j=1}^{M} n_{i j} \sigma_{j} .
$$

Since $\Gamma\left(-z_{j}\right)$ is analytic for $\operatorname{Re} z_{j}=\sigma_{j}<0$ and decreases exponentially with $\left|\operatorname{Im} z_{j}\right|=\left|y_{j}\right|,[18]$ :

$$
\lim _{|y| \rightarrow \infty}|\Gamma(\sigma+i y)| e^{\pi \frac{|y|}{2}}|y|^{1 / 2-\sigma}=\sqrt{2 \pi}
$$

the last integral is convergent. So is the first one in (III.11) if we can satisfy the supplementary conditions :

$$
\sum_{j=1}^{M} n_{i j} \sigma_{j}+\omega_{i}=\sum_{j=1}^{M}\left(n_{i j}-\frac{\omega_{i}}{\omega}\right) \sigma_{j}>0
$$


for $i=1, \ldots, l-1$. Let us define the convex domain:

$$
D_{h}=\left\{\sigma \mid \begin{array}{l}
\sum_{j=1}^{M} \sigma_{j}=-\omega ; \quad \sigma_{j}<0, \quad j=1, \ldots, M \\
\sum_{j=1}^{M}\left(n_{i j}-\frac{\omega_{i}}{\omega}\right) \sigma_{j}>0, \quad i=1, \ldots, l-1
\end{array}\right\} .
$$

Thus, if $D_{h}$ is not empty, absolute convergence is ensured and we may write what we call MM (Multiple Mellin) - representation of integral (III.8):

$$
G_{h}=\int_{\sigma \in D_{h}} M\left(z_{j}\right)
$$

with

$$
\begin{aligned}
M\left(z_{j}\right) & =\left(\prod_{j=1}^{M} \Gamma\left(-z_{j}\right)\right) \int_{0}^{1}\left(\prod_{i=1}^{l-1} \beta_{i}^{\phi_{i}-1} d \beta_{i}\right) u^{\omega-D / 2} \prod_{j=1}^{M} v_{j}^{z_{j}}, \\
\phi_{i} & =\sum_{j=1}^{M}\left(n_{i j}-\frac{\omega_{i}}{\omega}\right) z_{j} .
\end{aligned}
$$

(In the special case where $V$ is FINE and $\omega_{i}-\omega n_{i j_{h}}>0, D_{h}$ is not empty since it contains points $\sigma$ with all $\sigma_{j}$ slightly smaller than 0 , except $\sigma_{j_{h}}$ taken slightly larger than $-\omega$.) More generally $D_{h}$ gives a geometrical way to study convergence: if $D_{h}$ is not empty, $G_{h}$ is finite, and actually independent on the choosen point $\sigma$ in $D_{h}$, as can be seen by Cauchy theorem, since $M\left(z_{j}\right)$ is analytic in this domain.

But we prove in Appendix A the converse assertion: convergence of integral (III.8), which is implied by the existence of the Feynman amplitude before scaling, is sufficient to prove that $D_{h}$ is not empty. Therefore we may write an absolutely convergent MM-representation in each sector for any finite euclidean Feynman amplitude. We shall see in the following section how this representation induces an asymptotic expansion in the scaling parameter.

\section{Remark on Ultra-violet Divergent Graphs}

The case where zeros of $U$ induce ultra-violet divergences can be reduced to the convergent case along the following lines: in the $\alpha$-parametric integral representation, these divergences are renormalized by Taylor subtractions. But the remainder of the Taylor expansion may be written as in [16]:

$$
\left(1-\tau^{n}\right) f(x)=\int_{0}^{1} d \xi \frac{(1-\xi)^{n+\mu}}{(n+\mu) !}\left(\frac{d}{d \xi}\right)^{n+\mu+1}\left[\xi^{\mu+\varepsilon} f(\xi x)\right] .
$$

By regrouping the nests which belong to the same equivalence class as explained in Ref. [16], we obtain in each sector and for each equivalence class, a finite sum of convergent integrals which are exactly of the same type as in (III.8), provided that the various $\xi$-variables are simply renamed as supplementary 
$\beta$-variables. Thus we have again an MM-representation, with only some unessential new features: the number of $\beta$-variables becomes larger, and the splitting into FINE pieces $\sum_{j=1}^{M} v_{j} \prod_{i} \beta_{i}^{n_{i}}$ has to be performed only after renormalization. In the following we keep the notations corresponding to the convergent case.

\section{Asymptotic Expansion}

Starting with the MM-representation (III.15), if we now scale some invariants and/or masses by $\lambda$ we get:

$$
G_{h}(\lambda)=\int_{\sigma \in D_{h}} \lambda_{j} \sum_{j=1}^{N} z_{j} M\left(z_{j}\right)
$$

with $M\left(z_{j}\right)$ given in (III.16), where $u^{\omega-D / 2} \prod_{j=1}^{M} v_{j}^{z_{i}}$ has a simultaneous Taylor expansion in the $\beta$ 's, with non-vanishing constant term. When $\operatorname{Re} z=\sigma \in D_{h}, M\left(z_{j}\right)$ is analytical and the integral is absolutely convergent. So we may give a first bound:

$$
G_{h}(\lambda)<\text { Const }^{\dagger} \lambda^{p_{h}+\varepsilon},
$$

where $\varepsilon$ is positive and arbitrarily small, and

$$
p_{h}=\operatorname{Inf}_{D_{h}} \sum_{j=1}^{N} \sigma_{j} .
$$

Actually $p_{h}$ is always finite since in $D_{h}$ :

$$
\sum_{j=1}^{N} \sigma_{j}=-\omega-\sum_{j=N+1}^{M} \sigma_{j}>-\omega .
$$

We note that $p_{h}$ is rational, as given by intersection of hyperplanes with rational coefficients.

Next we perform an analytic continuation of $M\left(z_{j}\right)$ up to points $z^{\prime}$ where $\psi_{0}\left(\sigma^{\prime}\right) \equiv \sum_{j=1}^{N} \sigma_{j}^{\prime}-p_{h}$ becomes negative. Let us relabel the bounds of $D_{h}$ by:

$$
D_{h}\left\{\sigma \mid \psi_{v}(\sigma)>0, v=1, \ldots, M+l-1\right\},
$$

where the complete list of $\psi_{v}$ 's, in the space of independent variables $\sigma_{1}, \ldots, \sigma_{M-1}$, is :

$$
\begin{aligned}
& \psi_{v}(\sigma) \equiv-\sigma_{v}, \quad v=1, \ldots, M-1 \\
& \psi_{v}(\sigma) \equiv \sigma_{1}+\ldots+\sigma_{M-1}+\omega, \quad v=M \\
& \psi_{v}(\sigma) \equiv \sum_{j=1}^{M-1}\left(n_{i j}-n_{i M}\right) \sigma_{j}+\omega_{i}-n_{i M} \omega, \quad v=M+i, \quad i=1, \ldots, l-1 .
\end{aligned}
$$


Of course several $\psi_{v}$ 's may be identical. Each singularity $\frac{1}{\psi_{v}(z)}$ may be easily isolated in the integrand:

- for $v=1, \ldots, M$ by $\Gamma\left(-z_{j}\right)=-\frac{1}{z_{j}} \Gamma\left(-z_{j}+1\right)$,

- for $v=M+i$ by integrating over $\beta_{i}$ with the first-order term in the Taylor expansion of $u^{\omega-D / 2} \prod v_{j}^{z_{j}}$. The integrand $M(z)$ becomes :

$$
M(z)=\left(\prod_{v} \frac{1}{\psi_{v}(z)}\right) M^{\prime}(z)
$$

where $M^{\prime}(z)$ is now analytical in a bigger domain:

$$
\psi_{v}(\sigma)+1>0 \quad v=1, \ldots, M+l-1 .
$$

But since $\psi_{0}(\sigma) \equiv \sum_{j=1}^{N} \sigma_{j}-p_{h}$ is positive in $D_{h}$ and vanish only on its bound, it must be a linear form which belongs to the convex space generated by the $\psi_{v}$ 's ; there exist (generally not unique) non-negative $d_{v}$ 's, with:

$$
\psi_{0} \equiv \sum_{\nu=1}^{M+l-1} d_{\nu} \psi_{\nu}
$$

Thus

$$
\prod_{v} \frac{1}{\psi_{v}} \equiv \frac{1}{\psi_{0}} \sum_{v}\left(d_{v} \prod_{v^{\prime} \neq v} \frac{1}{\psi_{v^{\prime}}}\right)
$$

For a given term in this sum, if there still exist non-negative coefficients $d_{v^{\prime}}^{\prime}$ such that:

$$
\psi_{0} \equiv \sum_{v^{\prime} \neq v} d_{v^{\prime}}^{\prime} \psi_{v^{\prime}}
$$

it means that $\psi_{0}(\sigma)$ remains positive in the bigger domain:

$$
\left\{\begin{array}{l}
\psi_{v}(\sigma)+1>0 \\
\psi_{v^{\prime}}(\sigma)>0, \quad v^{\prime}=1, \ldots, v-1, v+1, \ldots, M+l-1
\end{array}\right.
$$

and we write:

$$
\frac{1}{\psi_{0}} \prod_{v^{\prime} \neq v} \frac{1}{\psi_{v^{\prime}}} \equiv \frac{1}{\psi_{0}^{2}} \sum_{v^{\prime} \neq v}\left(d_{v^{\prime}}^{\prime} \prod_{v^{\prime \prime} \neq v, v^{\prime}} \frac{1}{\psi_{v^{\prime \prime}}}\right) .
$$

This procedure is repeated again and again, until we obtain:

$$
\prod_{v=1}^{M+l-1} \frac{1}{\psi_{v}} \equiv \sum_{E} \frac{C_{E}}{\psi_{0}^{q_{E}+1}} \prod_{v \in E} \frac{1}{\psi_{v}},
$$

where each $E$ is a subset of $\{1, \ldots, M+l-1\}$ such that $\psi_{0}$ does not belong to the convex space generated by the subset $\left\{\psi_{v}, v \in E\right\}$, which means that $\psi_{0}(\sigma)$ becomes 
negative somewhere in the domain $D_{E}$ :

$$
D_{E}=\left\{\sigma \mid \psi_{v}(\sigma)>0, v \in E ; \psi_{v}(\sigma)+1>0, v \notin E\right\} .
$$

In such a way we decompose the MM-representation as:

$$
\begin{aligned}
\mathrm{G}_{h}(\lambda) & =\sum_{E} \mathrm{c}_{E} \mathrm{G}_{h E}(\lambda) \\
G_{h E}(\lambda) & =\int_{\sigma \in D_{h}} \lambda^{\psi_{0}(z)+p_{h}} \frac{1}{\psi_{0}^{q_{E}+1}(z)} M_{E}(z),
\end{aligned}
$$

where $M_{E}(z)$ is now analytical in $D_{E}$ and

$$
\operatorname{Inf}_{D_{E}} \sum_{j=1}^{N} \sigma_{j}=p_{h}-\eta_{E} \text {. }
$$

$\eta_{E}$ being a strictly positive rational number.

By Cauchy theorem, we may displace the integration path up to a point $\sigma^{\prime}$ in $D_{E}$ where

$$
\sum_{j=1}^{N} \sigma_{j}^{\prime}=p_{h}-\eta_{E}+\varepsilon
$$

This gives :

$$
G_{h E}(\lambda)=\lambda^{p_{h}} \sum_{q=0}^{q_{E}} g_{p_{h} q} \ln ^{q} \lambda+H_{h E}(\lambda)
$$

with:

$$
g_{p_{h} q}=\frac{1}{q !\left(q_{E}-q\right) !} \int_{\substack{\sigma \in D_{E} \\ \psi_{0}(z)=0}} \nabla^{q_{E}-q} M_{E}(z)
$$

where $\boldsymbol{V}$ is the differential operator along any direction crossing the plane $\psi_{0}(z)=0$ in the positive direction, and:

$$
H_{h E}(\lambda)=\int_{\sigma^{\prime}} \lambda^{\psi_{0}(z)+p_{h}} \frac{1}{\psi_{0}^{q_{E}+1}(z)} M_{E}(z)<\operatorname{Const}^{t} \lambda^{p_{h}-\eta_{E}+\varepsilon} .
$$

Therefore the "leading-power" part of the expansion in the sector $h$ is determined. In the same way we can extract all singularities $\frac{1}{\psi_{v}(z)+n}$ from $M(z)$, and similarly determine the complete asymptotic expansion.

It may be noticed that the leading term of this expansion is:

$$
\lambda^{p_{\max }} \mid n^{q_{\max }} \lambda g_{p_{\max } q_{\max }},
$$

where

$$
g_{p_{\max } q_{\max }}=\sum_{\substack{\text { Sectors } h \text { such } \\ \text { that } p_{h}=p_{\max }}} \sum_{\substack{E \text { such } \\ \text { sut } q_{E}=q_{\max }}} \frac{c_{E}}{q_{\max } !} \int_{\substack{\sigma \in D_{E} \\ \psi_{0}(z)=0}} \mathrm{M}_{E}(z)
$$


is a sum of positive terms : no cancellation between different terms in a sector, nor between different sectors, can occur for the leading behaviour of $G(\lambda)$ (except in the case of ultra-violet divergent graphs, where differentiations respective to new variables $\xi$ may introduce negative terms).

\section{Feynman Amplitudes in Minkowskian Metric}

For physical amplitudes with minkowskian metric, each propagator is written as:

$$
\frac{i}{q^{2}-m^{2}+i \varepsilon}=\int_{0}^{\infty} d \alpha e^{i \alpha\left(q^{2}-m^{2}+i \varepsilon\right)}
$$

and integral representation (II.1) is replaced by:

$$
G=\lim _{\varepsilon \rightarrow 0} \int_{0}^{\infty} \prod_{i=1}^{l} d \alpha_{i} \frac{1}{U^{D / 2}} e^{-\varepsilon} \sum_{i=1}^{l} \alpha_{\imath}+i \frac{V}{U}
$$

with (II.3) replaced by:

$$
V=\sum_{2 \cdot T}\left(\prod_{i \notin 2 \cdot T} \alpha_{i}\right)\left(\sum_{2 \cdot T} p\right)^{2}-\left(\sum_{i=1}^{l} \alpha_{i} m_{i}^{2}\right) U,
$$

where now the invariants $\left(\sum_{2 \cdot T} p\right)^{2}$ are Lorentz invariants and $V$ has no positivity property. As before, we split the scaled $W$-part and the unscaled $R$-part of $V$ into FINE pieces $V_{j}, j=1, \ldots, M$. Integrating again over $\beta_{l}$ in each sector $h$ we get:

$$
G_{h}=\lim _{\varepsilon \rightarrow 0} \Gamma(\omega) \int_{0}^{1} \frac{\left(\prod_{i=1}^{l-1} \beta_{i}^{\omega_{i}-1} d \beta_{i}\right) u^{\omega-D / 2}}{\left[-i \sum_{j=1}^{M} v_{j} \prod_{i=1}^{l-1} \beta_{i}^{n_{t}}+\delta\right]^{\omega}}
$$

with :

$$
\delta=\varepsilon\left(1+\beta_{l-1}+\ldots+\beta_{l-1} \ldots \beta_{2} \beta_{1}\right) u \prod_{i=1}^{l-1} \beta_{i}^{L_{l}}
$$

(we know that $L_{i} \leqq \operatorname{Inf}_{j} n_{i j}$ since $\frac{V}{U}$ never becomes infinite). A particularly simple situation emerges if all pieces $V_{j}$ do not vanish for non-vanishing $\alpha$ 's, and have the same common sign (as an example, it is actually true for on mass-shell vertex graphs in quantum electrodynamics: see $\S 6$, Sect. VI). In this case indeed, after $\beta_{l}$-integration is performed, we may interchange the order of limit and integrations, and write:

$$
G_{h}=( \pm i)^{\omega} \Gamma(\omega) \int_{0}^{1} \frac{\left(\prod_{i=1}^{l-1} \beta_{i}^{\omega_{i}-1} d \beta_{i}\right) u^{\omega-D / 2}}{\left[\sum_{j=1}^{M}\left|v_{j}\right| \prod_{i=1}^{l-1} \beta_{i}^{n_{z_{j}}}\right]^{\omega}}
$$


where $u$ and $\left|v_{j}\right|$ again have upper and lower strictly positive real bounds. One way to check such an assertion, along the lines of our paper, is shown in Appendix B. Thus we are left again with an euclidean problem which may be handled exactly like before.

In any case, we could attribute the $M$ th part of $\varepsilon$ to each $i V_{j}$ and write:

$$
G_{h}=\lim _{\varepsilon \rightarrow 0} \int_{0}^{1}\left(\prod_{i=1}^{l-1} \beta_{i}^{\omega_{i}-1} d \beta_{i}\right) u^{\omega-D / 2} \int_{\sigma} \prod_{j=1}^{M}\left(\Gamma\left(-z_{j}\right) A_{j}^{z_{j}}\right),
$$

where

$$
A_{j}=\frac{\delta}{M}-i v_{j} \prod_{i=1}^{l-1} \beta_{i}^{n_{i j}}, \quad \operatorname{Re} A_{j}=\frac{\delta}{M}>0 \quad \text { and } \quad A_{j} \text { is FINE. }
$$

For strictly positive $\varepsilon, \delta$ remains positive and each $\left|\arg A_{j}\right|$ is bounded by a value $\theta(\varepsilon)<\frac{\pi}{2}$.

Absolute convergence is verified again and we may write an MM-representation:

$$
G_{h}=\lim _{\varepsilon \rightarrow 0} \int_{\sigma \in D_{h}} \prod_{j=1}^{M} \Gamma\left(-z_{j}\right) \int_{0}^{1}\left(\prod_{i=1}^{l-1} \beta_{i}^{\omega_{2}-1} d \beta_{i}\right) u^{\omega-D / 2} \prod_{j=1}^{M} A_{j}^{z_{j}} .
$$

This form however is not yet satisfactory since we cannot take $\varepsilon=0$ in each $A_{j}$ in order to exhibit relevant singularities. We must study carefully the absolute convergence of integrals like:

$$
\int_{\sigma} \Gamma(-z) \Gamma(z+\omega) A^{z} B^{-z-\omega}
$$

in the limit $\arg \left(\frac{A}{B}\right) \rightarrow \pm \pi$. This convergence can be deduced from integrations by parts, isolating threshold singularities. But we leave for a later paper a more complete study of this problem.

\section{Discussion and Examples of Applications}

1 .

First we note that the MM-representation (IV.1) can be used directly to find an asymptotic behaviour in $\lambda$ when $\lambda \rightarrow 0$. Indeed it is sufficient to work like in Sect. IV, but with

$$
p_{h}^{\prime}=\operatorname{Sup}_{D_{h}} \sum_{j=1}^{N} \sigma_{j} .
$$

The result is of course an expansion with increasing rational powers of $\lambda$, starting from $p_{\min }$, and integer powers of $\ln \lambda$. Actually, since each $D_{h}$ is a nonempty open domain,

$$
p_{h}^{\prime}>p_{h} .
$$


This ensures the existence of a simple Mellin transform (II.5) for each $G_{h}(\lambda)$. But $G(\lambda)$ itself has a simple Mellin transform only if :

$$
p_{\min }=\operatorname{Inf}_{h} p_{h}^{\prime}>p_{\max }=\operatorname{Sup}_{h} p_{h} .
$$

2.

Examples of direct applications of the techniques exposed in this paper are given in $\S \S 6$ and 7. These applications are performed in Hepp's sectors. Theoretically, we give one way to compute the coefficients $g_{p q}$ of the asymptotic expansion (I.2). But this method is rather lengthy: one first divides into $l$ ! sectors, then one has to study the relative positions of the hyperplanes $\psi_{v}(\sigma)=0$ and to determine the convex polyhedron $D_{h}$. Finally one must find $p_{h}$ and decompose $\psi_{0}$ into convex linear combinations of $\psi_{v}$ 's as many times as needed. Hopefully, for a given asymptotic problem, especially for the leading power terms, all these operations turn out to be simple, due to topological properties of Feynman graphs. Moreover in some cases, it can be sufficient to obtain results on the powers in the expansion, and then to use differential equations and symmetry properties, in order to sum the logarithms without computing explicitly their coefficients.

On the other hand, in order to interpret the coefficients and eventually to sum over all graphs the logarithms of the leading power terms, it would be better to express the coefficients in terms of subgraphs and reduced graphs. This is generally not apparent when the coefficients are computed in the sectors: it would be useful to have at one's disposal a formalism working directly in the $\alpha$-space representation, as it is the case with simple Mellin transform. Then one could obtain general results on the various problems mentioned in the introduction.

Thus, more important are the indirect applications, where our method justify certain manipulations of the $\alpha$-integrals in order to compute $g_{p q}$ in terms of Feynman-like integrals attached to subgraphs and reduced graphs. Such applications are commented in $\S \S 3$ and 4.

\section{Large Momenta Behaviour in $\phi^{4}$ Field Theory}

Given a graph $G$ of $\phi^{4}$ field theory and its Feynman amplitude $I_{G}(p, m)$. We scale all external momenta $p$ by $\lambda$ and we look for the coefficients $g_{p_{\max } q}$ of the asymptotic expansion when $\lambda \rightarrow \infty$. Such a problem was solved in Ref. [3]. It was shown there that the polynomials in $\alpha$ which determine the asymptotic behaviour are FINE, and consequently the single Mellin transform was sufficient to prove Theorem (I.2). The Mellin transform $M_{G}(x)$ is meromorphic with multiple poles at integer values $\Omega, \Omega-2, \Omega-4, \ldots$ ( $\Omega$ is the superficial degree of divergence of the graph $G$ ). It was shown that there exists an operator $R$ which acts directly on the $\alpha$-variables, and which analytically continues $M_{G}(x)$ beyond the first pole and extracts in the $\alpha$-variables all the structure of the residue at $x=\Omega$. We give here the result for non-exceptional momenta in Euclidean space:

$$
\begin{aligned}
I_{G}(\lambda p, m)= & \sum_{\mathscr{F}} \frac{\chi_{\mathscr{F}}}{\left(q_{\mathscr{F}}+\delta\right) !} \prod_{S \in \mathscr{F}}\left(2 \tilde{\beta}_{[S]}^{\chi \omega(s)}\right) \\
& \cdot \frac{d^{q_{F}}+\delta}{d x^{q_{F}}+\delta}\left\{\lambda^{x}(x-\Omega)^{\delta} \Gamma(-x) 2 \tilde{\gamma}_{[G]_{\mathscr{F}}}(x, p, m)\right\}_{x=\Omega}+O\left(\lambda^{\Omega-2}\right),
\end{aligned}
$$

where $\delta=+1$ if $\Omega \geqq 0$ and 0 otherwise. 
In (VI.4) we sum over all (possibly empty) forests $\mathscr{F}$ of $q_{\mathscr{F}}$ divergent generalized vertices different from $G$ itself. The functions $\tilde{\beta}$ and $\tilde{\gamma}$, and the numerical coefficient $\chi_{\mathscr{F}}$ may be found in Ref. [3]. It is important to note that these functions are attached to reduced graphs $[S]_{\mathscr{F}}$ (and $[G]_{\mathscr{F}}$ ) obtained by shrinking into points all the elements of $\mathscr{F}$ inside $S$ (and $G$ ). The factorization property observed in the result (VI.4) explains the infinite summation of logarithms over all graphs of a vertex function [12] and reconstructs a solution to the homogeneous Callan-Symanzik differential equation [19].

\section{Large s Behaviour of Four-Bodies Amplitudes in $\phi^{3}$ Field Theory}

Given an essentially planar graph $G$ of the four point vertex function in $\phi^{3}$ field theory, that is a graph the Feynman amplitude of which is only a function of $s$ and $t$. We wish to determine the large $s$ behaviour at fixed $t$. It is shown in Ref. [13] that the polynomial in $\alpha$ which determines the asymptotic regime is not FINE. The consequence is that a priori the $R$ operator used in $\S 3$ does not desingularize the single Mellin transform around the leading pole. This is why the example of $\S 7$ is performed in the sectors. We are presently working on the construction of a new operator acting in the $\alpha$-variables space which should be able to desingularize the single Mellin transform even when the polynomial is not FINE. A detailed knowledge of multiple Mellin transform techniques is needed for such a construction. Fortunately, as we show in Ref. [13], for a large class of amplitudes in $\phi^{3}$ field theory, and especially for all leading amplitudes (behaviour in $s^{-1}$ up to logarithms), the convex polyhedrons described in Sect. IV are such that despite the non-FINE character of the polynomial, the $R$ operator does desingularize the single Mellin transform. If we decompose the graph $G$ in two particles irreducible kernels $N_{i}$ in the $t$-channel, the leading graphs are found to be those graphs in which at least one kernel $N_{i}$ is a single rung. If $G$ contains $r$ kernels $N_{i}$ which are single rungs and if $J$ is a non-empty subset of these $r$ kernels with $v(J)$ single rungs, we have proved that

$$
\begin{aligned}
I_{G}(s, t) & =\sum_{J} \frac{(-g)^{2 v(J)}}{[v(J)-1] !} \frac{d^{v(J)-1}}{d x^{v(J)-1}}\left\{\Gamma(-x) e^{-i \pi x} S^{x}[\Gamma(x+2)]^{v(J)}\right. \\
& \left.\cdot m^{-2 v(J)(x+1)} \bar{M}_{[G / J]}\left(x, t, p_{i}^{2}, m, g\right)\right\}_{x=-1}+O\left(s^{-1-\eta}\right) .
\end{aligned}
$$

The function $\bar{M}_{[G / J]}$ is defined in Ref. [13] and is attached to the reduced graph obtained from $G$ when the $v(J)$ single rungs are shrinked into $v(J)$ points. This function factorizes into several parts and such a factorization as well as the general structure given in (VI.5) is the cause for a possible summation of all $\ln s$ over all leading graphs to give a behaviour of the form $\beta(t, m, g)\left(\frac{s}{m^{2}}\right)^{\alpha(t, m, g)}$ where $\alpha(t, m, g)$ and $\beta(t, m, g)$ are known as power series and described in Ref. [13].

A similar program is now in the process of being performed by us for $\phi^{4}$ field theory.

\section{Technical Comments on the General Method in Hepp's Sectors}

We now turn to the cases where the $R$ operator does not desingularize the single Mellin transform expressed in the $\alpha$-variables, as is the case for instance in the on 
mass-shell infrared limit of quantum electrodynamics or quantum chromodynamics. Presently, we have not yet found the algorithm in the $\alpha$-variables which replaces the $R$ operator and we are condemned to work in the sectors.

For any Feynman amplitude, in a sector $h\left(\alpha_{k_{1}} \leqq \alpha_{k_{2}} \leqq \ldots \leqq \alpha_{k_{l}}\right)$ the last $(l-1)$ linear forms $\psi_{v}, v=M+i$, are the powers of $\beta_{i}$ in $M_{h}(z)$ (see Sect. IV):

$$
\psi_{v}(z)=\sum_{j=1}^{M}\left(n_{i j}-\frac{\omega_{i}}{\omega}\right) z_{j},
$$

where $n_{i j}$ is the factorized power of $\beta_{i}$ in $V_{j}, \omega_{i}=i+\left(\omega-\frac{D}{2}\right) L_{i}, L_{i}$ is the factorized power of $\beta_{i}$ in $U$. Thus $n_{i j}$ and $L_{i}$ are also the degrees of vanishing of $V_{j}$ and $U$ when $\alpha_{k_{1}}, \ldots, \alpha_{k_{l}}$ go to zero linearly. Let us call $S_{i}$ the subgraph formed by the lines $k_{1}, \ldots, k_{i}$. Then, the problem is to find nested subgraphs $S_{i}$ such that the corresponding linear forms $\psi_{v}$, together with the linear forms $\left(-z_{j}\right)$, generate $\psi_{0}$ with positive coefficients: we call such a set of $S_{i}$ 's a "leading nest".

With this notation, the determination of the leading power term, in the asymptotic expansion, amounts to the research of the leading nests which are subsets of the same nest (corresponding to a given sector). Finally, one has to find how many times one can remove one $S_{i}$ in each leading nest, until one reaches minimal leading nests (leading nests not containing smaller leading nests): the number of such removals gives the maximal power of $\ln \lambda$.

\section{Infra-Red Limit for Electron-Photon Vertex}

As we said in the introduction, this problem has been studied by many authors [8$10]$, but the general result is not rigorously proved, due to the non-validity of power counting. We shall present elsewhere [20] our own results and proofs: the purpose of this paragraph is simply to illustrate our method by the examples of graphs in Figs. 1 and 2. We simplify again by considering only spinless particles with squared masses $m^{2}$ ("electron" lines) and $\frac{\mu^{2}}{\lambda}$ ("photon" lines). The external electron lines are on their mass-shell: $p_{1}^{2}=p_{2}^{2}=m^{2}, q^{2}=\left(p_{1}-p_{2}\right)^{2}<0$. Then for the graph in Fig. 1:

$$
\begin{aligned}
G\left(q^{2}, m^{2}, \frac{\mu^{2}}{\lambda}\right) & =\lambda G\left(\lambda q^{2}, \lambda m^{2}, \mu^{2}\right) \\
& =\lambda \lim _{\varepsilon \rightarrow 0} \int_{0}^{\infty} \frac{\prod_{i=1}^{3} d \alpha_{i}}{U^{2}} e^{-\varepsilon \sum_{i=1}^{3} \alpha_{i}+i \frac{\lambda W+R}{U}}
\end{aligned}
$$

Fig. 1 .

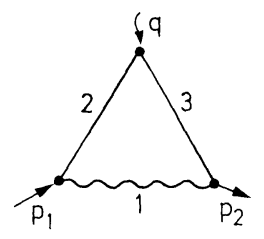


Fig. 2.

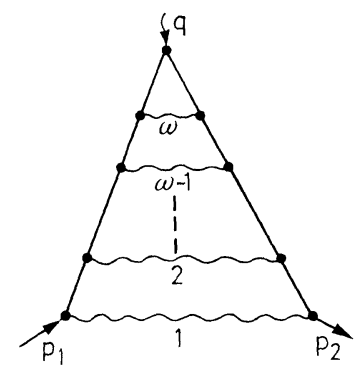

Fig. 3.

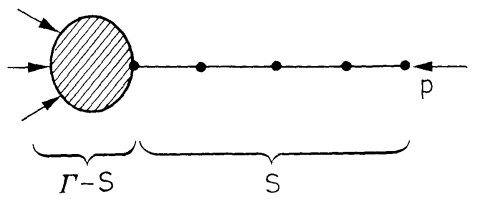

with:

$$
\begin{aligned}
& U=\alpha_{1}+\alpha_{2}+\alpha_{3} \\
& W=q^{2} \alpha_{2} \alpha_{3}-m^{2}\left(\alpha_{2}+\alpha_{3}\right)^{2} \\
& R=-\mu^{2} \alpha_{1}\left(\alpha_{1}+\alpha_{2}+\alpha_{3}\right) .
\end{aligned}
$$

We are in the simple situation treated in Sect. V since $V$ is a sum of negative monomials. Furthermore $W$ and $R$ are FINE polynomials. Thus a straightforward application of our technique allows to recover quickly the very well known result:

$$
G(\lambda)=A \ln \lambda+B+O\left(\lambda^{-\eta}\right),
$$

where $\eta$ is positive and $A=-i \int_{0}^{1} \frac{d \beta}{-q^{2} \beta+m^{2}(1+\beta)^{2}}$.

Let us consider now the graph given in Fig. 2. For this graph:

$\omega=l-2 L$ is equal to the number of photon lines.

$R=-\mu^{2}\left(\alpha_{1}+\ldots+\alpha_{\omega}\right) U$ is a FINE polynomial, but $W$ is no more FINE.

Among the properties of the $W$-function for a graph $\Gamma$ in Q.E.D., let us note the following ones:

i) If $i$ is a photon line:

$$
W_{\Gamma}=\alpha_{i} W_{\Gamma-\{i\}}+W_{\Gamma /\{i\}},
$$

where $\Gamma-\{i\}$ is the graph obtained by removing the line $i$ from $\Gamma, \Gamma /\{i\}$ is the graph obtained by reducing the line $i$ to a point.

ii) If $S$ is a chain of electron lines linked to an external vertex as in Fig. 3, and if the external electron is on its mass-shell, $p^{2}=m^{2}$ :

$$
W_{\Gamma}=W_{\Gamma-S},
$$

where $\Gamma-S$ is obtained by removing the chain $S$ from $\Gamma$.

Using these two identities, we decompose $W$ into $\omega$ FINE pieces:

$W=\sum_{j=1}^{\omega}\left(\prod_{i=1}^{j-1} \alpha_{i}\right) W_{\Gamma_{j}}$ 
Fig. 4.
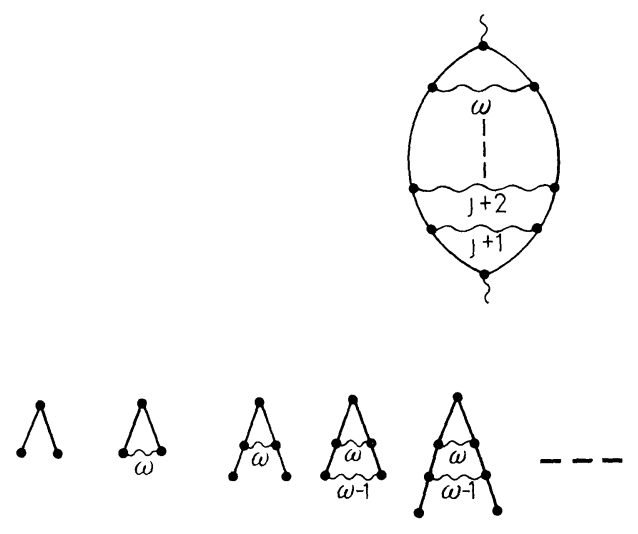

Fig. 5.

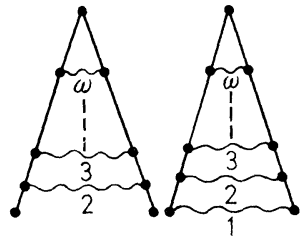

where $\Gamma_{j}$ is given in Fig. 4 and obtained by:

- removing the photon lines $i=1, \ldots, j-1$,

- removing then the external electron chains,

- reducing to a point the photon line $i=j$.

Again each $W_{j}$ is a FINE polynomial with only negative coefficients, and we recover the simple situation of Sect. V.

With this splitting of $W$ for the graph of Fig. 2, we determined all leading nests [20]. The leading power is $p_{\max }=0$ (only logarithmic divergences). As an example, the leading nest giving the highest power of $\ln \lambda$ is given in Fig. 5. It contributes to the leading term in the asymptotic expansion:

$$
G(\lambda)=\frac{A^{\omega}}{\omega !} \ln ^{\omega}(\lambda)+O\left(\ln ^{\omega-1} \lambda\right),
$$

where $A$ is the same quantity as given in (VI.7): this is not surprising since we expect exponentiation of infrared divergencies. But the application of such methods to the whole set of vertex graphs will give, in our opinion, the first rigorous proof of these old results.

\section{Example of Infinite Energy Limit}

As another illustration of our method, we indicate here how to find the $s \rightarrow \infty$ behaviour of the particular graph given in Fig. 6: one may verify that $R$ is FINE,

Fig. 6.

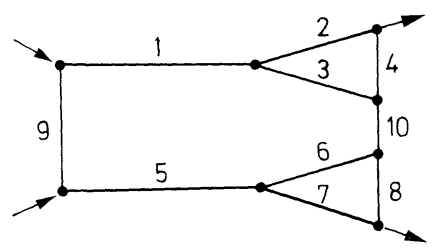


and $W$ may be split into $N=4$ FINE pieces

$$
W=s \sum_{j=1}^{4} V_{j}
$$

with

$$
\begin{aligned}
& V_{1}=\alpha_{1} \alpha_{5}\left(\alpha_{2}+\alpha_{3}+\alpha_{4}\right)\left(\alpha_{6}+\alpha_{7}+\alpha_{8}\right) \\
& V_{2}=\alpha_{1} \alpha_{6} \alpha_{7}\left(\alpha_{2}+\alpha_{3}+\alpha_{4}\right) \\
& V_{3}=\alpha_{2} \alpha_{3} \alpha_{5}\left(\alpha_{6}+\alpha_{7}+\alpha_{8}\right) \\
& V_{4}=\alpha_{2} \alpha_{3} \alpha_{6} \alpha_{7},
\end{aligned}
$$

where the lines are numbered as in Fig. 6. One of the leading terms of the expansion in $s$ is found in the sector : $\alpha_{1} \leqq \alpha_{2} \leqq \alpha_{3} \leqq \ldots \leqq \alpha_{10}$ and the relevant linear forms come from subgraphs $S_{i}$ for $i=1, \ldots, 8$ :

$$
\begin{aligned}
& \psi_{M+1}=z_{1}+z_{2}+1 \\
& \psi_{M+3}=z_{1}+z_{2}+2 z_{3}+2 z_{4}+3 \\
& \psi_{M+5}=2 z_{1}+z_{2}+2 z_{3}+z_{4}+3 \\
& \psi_{M+7}=2 z_{1}+3 z_{2}+2 z_{3}+3 z_{4}+5 \\
& \psi_{M+2}=\psi_{M+4}=\frac{1}{2} \psi_{M+6}=\frac{1}{2} \psi_{M+8}=z_{1}+z_{2}+z_{3}+z_{4}+2=\psi_{0} .
\end{aligned}
$$

The minimal leading nests are:

$$
\begin{array}{lll}
\mathscr{N}_{1}=\left\{\mathrm{S}_{1}, \mathrm{~S}_{3}\right\}, & \mathscr{N}_{2}=\left\{\mathrm{S}_{5}, \mathrm{~S}_{7}\right\}, & \mathscr{N}_{3}=\left\{\mathrm{S}_{2}\right\}, \\
\mathscr{N}_{4}=\left\{\mathrm{S}_{4}\right\}, & \mathscr{N}_{5}=\left\{\mathrm{S}_{6}\right\}, & \mathscr{N}_{6}=\left\{\mathrm{S}_{8}\right\}
\end{array}
$$

with the notations of $\S 5$, and we find for the amplitude an expansion:

$$
G(s)=g_{-2,5} s^{-2} \ln ^{5} s+O\left(s^{2} \ln ^{4} s\right) .
$$

In the same way, one could rigorously extract the complete expansion. Actually for this graph, the partial result (VI.13) was already known and may be found for instance in a paper by Tiktopoulos [4].

Acknowledgements. We thank for help and discussions several physicists from the Centre de Physique Thèorique at Ecole Polytechnique, especially J. Lascoux, and from D. Ph. T. at Saclay, especially F. David.

\section{Appendix A}

Let $G_{h}$ be an integral like (III.8):

$$
G_{h}=\Gamma(\omega) \int_{0}^{1} \frac{\left(\prod_{i=1}^{l-1} \beta_{i}^{\omega_{i}-1} d \beta_{i}\right) u^{\omega-D / 2}}{\left[\sum_{j=1}^{M} v_{j} \prod_{i=1}^{l-1} \beta_{i}^{n_{\imath j}}\right]^{\omega}},
$$


where $\omega, \omega_{i}$ are strictly positive integers or half-integers,

$n_{i j}$ are arbitrary non-negative integers,

$u, v_{j}$ have upper and lower strictly positive real bounds.

Definition. We call "generalized power-counting condition" the following property:

$$
\operatorname{Inf}_{j} \sum_{i=1}^{l-1} r_{i}\left(n_{i_{j}}-\frac{\omega_{i}}{\omega}\right)<0
$$

for any choice of real $r_{i}$ 's, $r_{i} \geqq 0, i=1, \ldots, l-1$, the $r_{i}$ 's being not all vanishing. (The naive power counting is only for $r_{i}=0$ or 1 .)

Theorem. The following three propositions are equivalent:

1) $G_{h}$ is a convergent integral.

2) The generalized power-counting condition is true.

3) Domain $D_{h}=\left\{\sigma \mid \sum_{j=1}^{M} \sigma_{j}=-\omega ; \sigma_{j}<0 ; \sum_{j=1}^{M}\left(n_{i j}-\frac{\omega_{i}}{\omega}\right) \sigma_{j}>0\right\}$ is not empty.

Proof. First we know that 3) implies 1) as shown in Sect. III by the MMrepresentation. Furthermore it is clear that 1) implies 2): we may take new variables by $\beta_{i}=\gamma_{i}^{r_{t}}$ for any subset of variables; then convergence of $G_{h}$ implies at least superficial convergence of the sub-integration over $\gamma$-variables.

Finally we achieve the proof by showing that 2) implies 3). Let us introduce the notations:

- closed first "quadrant": $\bar{Q}=\left\{\boldsymbol{r} \neq \mathbf{0} \mid r_{i} \geqq 0, i=1, \ldots, l-1\right\}$;

- open last "quadrant": $-Q=\left\{\boldsymbol{t} \neq \mathbf{0} \mid t_{i}<0, i=1, \ldots, l-1\right\}$;

- open convex cone: $C=\left\{\boldsymbol{a} \neq \mathbf{0} \mid\right.$ there exist $\tau_{j}>0$ with $\left.a_{i}=\sum_{j=1}^{M}\left(n_{i j}-\frac{\omega_{i}}{\omega}\right) \tau_{j}\right\}$;

- closed dual of $C: \bar{C}^{\prime}=\left\{\boldsymbol{s} \neq \mathbf{0} \mid \sum_{i=1}^{l-1} s_{i}\left(n_{i j}-\frac{\omega_{i}}{\omega}\right) \geqq 0\right.$ for every $\left.j\right\}$.

We have also: $C=$ open dual of $\bar{C}^{\prime}=\left\{\boldsymbol{a} \neq \mathbf{0} \mid \sum_{i=1}^{l-1} a_{i} s_{i}>0\right.$ for any $\left.\boldsymbol{s} \in \bar{C}^{\prime}\right\}$.

Proposition 2) means that $\bar{C}^{\prime} \cap \bar{Q}$ is empty. Thus by Hahn-Banach lemma, there exists a plane separating these two convex closed cones: there exists $\boldsymbol{u}$ such that $l-1$

$\sum_{i=1} u_{i} s_{i}>0$ for any $\boldsymbol{s} \in \overline{\boldsymbol{C}}^{\prime}$, which implies $\boldsymbol{u} \in C$;

(1)

$\sum_{i=1} u_{i} r_{i}<0$ for any $\boldsymbol{r} \in \bar{Q}$, which implies $\boldsymbol{u} \in-Q$.

Now since $(-Q) \cap C$ is not empty, there exist $\tau_{j}>0$ with

$$
\sum_{j=1}^{M} \tau_{j}\left(n_{i j}-\frac{\omega_{i}}{\omega}\right)<0, \quad i=1, \ldots, l-1
$$

or with $\sigma_{j}=-\tau_{j}$ :

$$
\sigma_{j}<0, \quad \sum_{j=1}^{M}\left(n_{i j}-\frac{\omega_{i}}{\omega}\right) \sigma_{j}>0, \quad i=1, \ldots, l-1
$$

and the last condition, $\sum_{j=1}^{M} \sigma_{j}=-\omega$, is realized by global normalization. 


\section{Appendix B}

Let $G$ be a Feynman integral in minkowskian metric:

$$
\begin{aligned}
G & =\lim _{\varepsilon \rightarrow 0} G(\varepsilon) \\
G(\varepsilon) & =\int_{0}^{\infty}\left(\sum_{i=1}^{l} d \alpha_{i}\right) \frac{1}{U^{D / 2}} e^{-\varepsilon \sum_{i=1}^{l} \alpha_{i}+i \frac{V}{U}}=\sum_{h} G_{h}(\varepsilon)
\end{aligned}
$$

with $G=\sum_{h} G_{h}$ and $G_{h}=\lim _{\varepsilon \rightarrow 0} G_{h}(\varepsilon)$.

Theorem. Let us assume that $G(\varepsilon)$ and $G$ exist (for ultra-violet divergent graphs, we assume that $G_{h}(\varepsilon)$ and $G_{h}$ exist for all $h$, after renormalization). If there exists a splitting of $V$ into FINE pieces $V_{j}$, such that:

no $V_{j}$ vanishes for non-vanishing $\alpha$ 's,

- all $V_{j}^{\prime}$ 's keep the same common sign,

then we may interchange in each sector the limit $\varepsilon \rightarrow 0$ and the $\beta$-integrations.

Proof. In each sector, we have an integral like (V.3), where the different terms in the denominator take values not in the half but only in a quarter of the complex plane, due to the common sign of the $V_{j}$ 's. By factorizing $e^{i \theta}, \theta= \pm \frac{\pi}{4}$, we write:

$$
G_{h}(\varepsilon)=e^{i \omega \theta} \Gamma(\omega) \int_{0}^{1} \frac{\left(\prod_{i=1}^{l-1} \beta_{i}^{\omega_{i}-1} d \beta_{i}\right) u^{(\nu-D / 2}}{\left[e^{-i \theta} \sum_{j=1}^{M}\left|v_{j}\right| \prod_{i=1}^{l-1} \beta_{i}^{n_{i j}}+e^{i \theta} \delta\right]^{\omega \omega}} .
$$

Now each term in the denominator has a phase equal to $\pm \frac{\pi}{4}$ : we may write an absolutely convergent MM-representation with $M+1$ pieces, and simply use our method for the particular limit $\varepsilon \rightarrow 0$. The MM-representation of $G_{h}(\varepsilon)$ is valid in the domain :

$$
\Delta_{h}=\left\{\sigma \mid \begin{array}{l}
\sum_{j=1}^{M+1} \sigma_{j}=-\omega ; \quad \sigma_{j}<0, \quad j=1, \ldots, M+1 \\
\sum_{j=1}^{M+1}\left(n_{i j}-\frac{\omega_{i}}{\omega}\right) \sigma_{j}>0, \quad i=1, \ldots, l-1
\end{array}\right\},
$$

where by convention $n_{i, M+1}=L_{i}$. Therefore $G_{h}(\varepsilon)$ has an asymptotic expansion with powers of $\ln \varepsilon$ and increasing powers of $\varepsilon$ when $\varepsilon \rightarrow 0$. Furthermore, in the case of ultra-violet convergent graphs, no cancellation occurs for the leading term, as remarked at the end of Sect. IV. Now the existence of a non-vanishing amplitude, in the limit $\varepsilon \rightarrow 0$, implies : $p_{\min }=0 ; q_{\max }\left(p_{\min }\right)=0$ that is :

$$
G_{h}(\varepsilon)=g_{00}+O\left(\varepsilon^{\eta}\right), \quad \eta \text { positive } .
$$

But $\psi_{0}(z)=-z_{M+1}=\sum_{j=1}^{M} z_{M}+\omega$ is a singularity effectively present in $\Gamma\left(-z_{M+1}\right)$. For the absence of $\ln \varepsilon$ in the leading term, it must be a simple polar variety: $\psi_{0}(z)$ no more belongs to the convex space generated by the $\psi_{v}(z)$ 's, $v \neq M+1$. In other 
words, there must exist points $\sigma$ in:

$$
D_{h}=\left\{\sigma \mid \begin{array}{l}
\sum_{j=1}^{M} \sigma_{j}=-\omega ; \quad \sigma_{j}<0, \quad j=1, \ldots, M \\
\sum_{j=1}^{M}\left(n_{i j}-\frac{\omega_{i}}{\omega}\right) \sigma_{j}>0, \quad i=1, \ldots, l-1
\end{array}\right\}
$$

which expresses nothing but convergence of the integral (V.4). Moreover $g_{00}$ in (B.3) is given by (IV.18) and equal to the integral (V.4).

\section{References}

1. Weinberg, S.: Phys. Rev. 118, 838 (1960)

2. Fink,J.P.: J. Math. Phys. 9, 1389 (1968)

3. Bergère,M.C., Lam,Y.M.P.: Commun. math. Phys. 39, 1 (1974); Asymptotic expansion of Feynman amplitudes, Part II. The divergent case. Preprint, Freie Universität Berlin HEP (May 74/9)

4. Federbush, P.G., Grisaru, M.T.: Ann. Phys. 22, 263 (1963)

Bjorken, J.D., Wu, T.T.: Phys. Rev. 130, 2566 (1963)

Tiktopoulos, G.: Phys. Rev. 131, 480, 2373 (1963)

Trueman, T.L., Yao, T.: Phys. Rev. 132, 2741 (1963)

Polkinghorne, J.C.: J. Math. Phys. 4, 503, 1396 (1963)

Efremov, A.V.: Preprints R. 1242 and R. 1305 Joint Institute for Nuclear Research (1963)

5. Zavyalov, O.I. : Soviet Phys. JETP 20, 736 (1965)

Zavyalov, O.I., Stepanov, B. M.: Soviet J. Nucl. Phys. 1, 658 (1965)

6. Mc Coy, B.M., Wu, T.T.: Phys. Rev. D 12, 3257 (1975); 13, 1076 (1976)

7. Eden, R.J., Landshoff, P.V., Olive, D.I., Polkinghorne, J.C.: The analytic $S$-matrix. Cambridge: Cambridge University Press 1966

8. Yennie, D. R., Frautschi, S.C., Suura, H.: Ann. Phys. 13, 379 (1961)

9. Cvitanović, P., Kinoshita, T.: Phys. Rev. D 10, 3978, 3991 (1974)

10. Grammer, G.,Jr., Yennie, D. R.: Phys. Rev. D 8, 4332 (1973) Korthals Altes, C.P., de Rafael, E.: Nucl. Phys. B 106, 237 (1976)

11. Carrazone, J.J., Poggio, E.R., Quinn, H.R.: Phys. Rev. D 11, 2286 (1975) and: Errata and comments. Harvard Preprint (1975)

Cornwall, J.M., Tiktopoulos, G. : Phys. Rev. Letters 35, 338 (1975)

Kinoshita, T., Ukawa, A.: Preprint, Cornell L.N.S-322 (Nov. 1975)

Korthals Altes, C.P., de Rafael, E.: Phys. Lett. 62B, 320 (1976)

Cvitanović, P.: Phys. Rev. Letters 37, 1528 (1976)

12. Bergère, M.C., Bervillier, C.: To be published

13. Bergère, M.C., Gilain, C.: J. Math. Phys. 19, 1495 (1978)

14. Vartchenko, A.N.: Functional analysis and its applications 10, 13 (1976)

15. Hepp, K.: Commun. math. Phys. 2, 301 (1966)

16. Bergère, M.C., Zuber, J. B. : Commun. math. Phys. 35, 113 (1974)

Bergère, M.C., Lam, Y.M.P.: J. Math. Phys. 17, 1546 (1976)

17. Gradshteyn, I.S., Ryzhik, I.M.: Table of integrals, series, and products, p. 657. New York: Academic Press 1965

18. Bateman, H.: Higher transcendental functions, Vol. I, p. 47. New York: McGraw-Hill 1953

19. Callan, C. G. : Phys. Rev. D 2, 1541 (1970)

Symanzik, K.: Commun. math. Phys. 18, 227 (1970)

20. Malbouisson, A.P.C.: Doctoral Thesis (in preparation)

Communicated by R. Stora

Received October 8, 1977; in revised form March 20, 1978 